\title{
PART ORIENTATION AND SEPARATION TO REDUCE PROCESS COSTS IN ADDITIVE MANUFACTURING
}

\author{
Reichwein, Jannik; \\ Kirchner, Eckhard \\ Technische Universität Darmstadt
}

\begin{abstract}
Additive manufacturing offers great potential in geometric design through the layer-by-layer production of components. This is often used in the development of additively manufactured components to make components lighter. An even greater reduction in mass is possible if several components are combined into a more complex component. However, as complexity increases, so do the manufacturing costs, due to a higher demand for supporting structure, reworking and longer production time. Especially for complex components, which make poor use of the space available in the additive manufacturing system, component separation can be a useful way of reducing manufacturing costs. Therefore, a procedure for automated component separation is presented, which determines an optimal cutting plane with respect to the manufacturing costs. The presented procedure is evaluated using two exemplary components where a reduction of manufacturing costs up to $54 \%$ could be achieved.
\end{abstract}

Keywords: Additive Manufacturing, Design for Additive Manufacturing (DfAM), Part separation, Part consolidation, Product architecture

\section{Contact:}

Reichwein, Jannik

Technische Universität Darmstadt

Produktentwicklung und Maschinenelemente

Germany

reichwein@pmd.tu-darmstadt.de 


\section{INTRODUCTION}

The demand for individualized products and for lightweight designs results in a great economic importance of additive manufacturing (AM) in production today. This is primarily due to the advantages that AM can offer compared to conventional manufacturing processes. These include great freedom in geometric design and the individualization of components through cost-effective production of small quantities (Gibson et al., 2015). The technical possibilities and potentials of additive manufacturing are therefore an important competitive factor for companies. Nevertheless, only a few industries, such as medical technology or aerospace, are using additive manufacturing for the manufacturing of end products and a broader application is lacking. (Wohlers, 2018)

For that reason, there are great efforts in industry and research to achieve a wider dissemination of additive manufacturing. While the productivity of the manufacturing processes and the variety of materials that can be processed is growing, the manufacturing systems are becoming cheaper. Additional potentials can be raised by using the potentials of additive manufacturing in the design of components. Therefore, it is necessary to overcome the existing limitations of additive manufacturing and to manufacture more cost-effectively overall. A decisive limitation is the orientation of the components during the manufacturing process, which affects the quality of the functional surfaces and the amount of support structure. Especially for complex components the orientation of the components is not trivial and leads to the need for many supporting structures. This increases the costs for material and leads to a longer process duration. Furthermore, a compromise must be made between the orientation of different functional surfaces, which increases the post-processing effort.

In addition to these limitations, there is a severe restriction of the available space in the building chamber of many additive manufacturing systems. In order to address these challenges, a procedure for component separation and orientation in the building space for Laser Powder Bed Fusion (L-PBF) of metal components is presented. This allows a more cost-effective production of complex components and the production of components that exceed the building space.

\section{STATE OF THE ART}

The possibilities and restrictions of the different manufacturing processes diverge significantly from each other. To take this into account already during product development, there are methods that are summarized under the generic term Design for Manufacturing (DfM). These aim to provide knowledge to improve the design of a product regarding manufacturability. AM offers new potentials and opportunities but also causes limitations compared to conventional processes (Gibson, 2015). Hence, there are DfM approaches especially for additive manufacturing that are summarized by the term Design for Additive Manufacturing (DfAM).

In recent years, there has been a great interest in research into DfAM. This includes adapted models of the design process that suits the requirements for designing products for additive manufacturing (Kranz, 2017; Kumke, 2018) as well as a number of individual methods that can be divided into restrictive and opportunistic methods (Laverne et al., 2015). Restrictive methods limit the solution space, ensure manufacturability and are often presented in the form of design guidelines (Adam, 2015; Kranz et al., 2015). Opportunistic methods expand the solution space and support the designer in finding new solutions. These include different types of solution databases (Reichwein et al., 2019) or for example adaptations of the TRIZ methodology to additive manufacturing (Schulte et al., 2020).

Due to the strongly differing possibilities of AM, not only the design of individual components should be adapted, but the entire product architecture. In this context, product architecture describes the product structure as the physical structure and the functional structure of a product combined and relates their elements to each other (Krause and Gebhardt, 2018). The variation of product architecture is beneficial for an adaptive design of existing products that are to be manufactured additively, in order to use the potentials of additive manufacturing and to meet the manufacturing restrictions. For example, there are methods for the combination of several components to form a single more complex component, which is called part consolidation (Kim and Moon, 2020; Nie et al., 2020). However, the separation of components for additive manufacturing is much less frequently discussed in the literature on DfAM and existing approaches usually only provide for the separation of components that are otherwise too large for the building space of an additive manufacturing system (Deka und Behdad, 2019; Oh et al., 2018). 


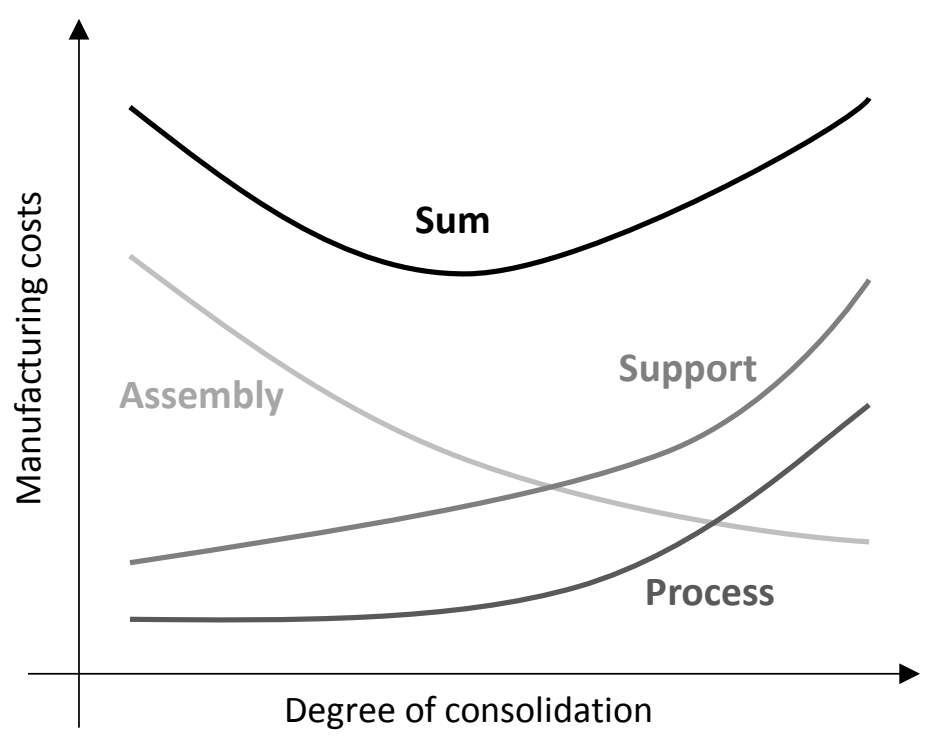

Figure 1: Degree of consolidation based on (Nie et al., 2020)

The product architecture can be described by the degree of consolidation, whereby a high degree of consolidation corresponds to an integral design and a low degree to a differential design. (Nie et al., 2020) has plotted the relationship of manufacturing costs over the degree of consolidation of the components (see Figure 1). The assembly costs decrease with increasing consolidation of the components because fewer assembly operations are necessary. However, the components become more complex with increasing consolidation and therefore require more supporting structures. This in turn leads to higher material consumption and more reworking. As can be seen in figure 1, this results in an optimum regarding manufacturing costs for many components with respect to the degree of consolidation. This optimum is dependent on the geometry and the required part count of the component and not necessarily found with a high degree of consolidation. (Nie et al., 2020)

\section{PROBLEM STATEMENT}

Separating components for additive manufacturing offers several potentials. Especially for geometrical complex components many supporting structures are necessary. These are required to attach the parts to the build platform during the manufacturing process and depending on the type of AM process to conduct thermal energy into the build platform. Support structure leads to a higher material consumption, longer process duration and more reworking effort, because the supporting structures must be removed from the component after production. By separating a complex component into individual parts, the volume of supporting structures can be significantly reduced. This results from the independent orientation of each individual part in the building space.

A further advantage of component separation is therefore the production of functional surfaces, which usually require a certain orientation in the building space in order to meet the corresponding surface requirements. Often, however, the orientations of several functional surfaces on a complex component are contradictory, which means that a compromise must be made in the orientation of the complete component. By separating the complex component an independent positioning of the individual parts is possible in order to minimize the post-processing of functional surfaces.

A further decisive aspect is the size of the component, which must not exceed the maximum dimensions of the building space of the additive manufacturing system. By separating components that are too large, it is possible to manufacture the single parts and join them afterwards. In addition, the independent positioning of the components enables a better use of the available building space. The optimal orientation regarding manufacturing costs is dependant to many factors, like the part count to be manufactured, the material, and the size of the building space.

In the state of the art, however, mainly methods for the consolidation of components are presented, whereby the optimum described in Figure 1 cannot be realized. The contribution of this paper is therefore a procedure for component separation considering the orientation of the parts in the building space in order to reduce the manufacturing costs of additively manufactured components. Initially, the 
approach presented here is limited to the manufacturing of metallic components using the L-BPF process. A transfer of the approach to other additive manufacturing processes seems possible.

\section{COST MODEL FOR ADDITIVE MANUFACTURING}

Component separation can be performed at various points on the component. In order to compare and evaluate the different separation variants, the manufacturing costs of the resulting parts are used in this approach. Therefore, a calculation of the manufacturing costs is necessary to choose a suitable separation variant. Influencing factors on the manufacturing costs for the L-PBF are shown in Figure 2.

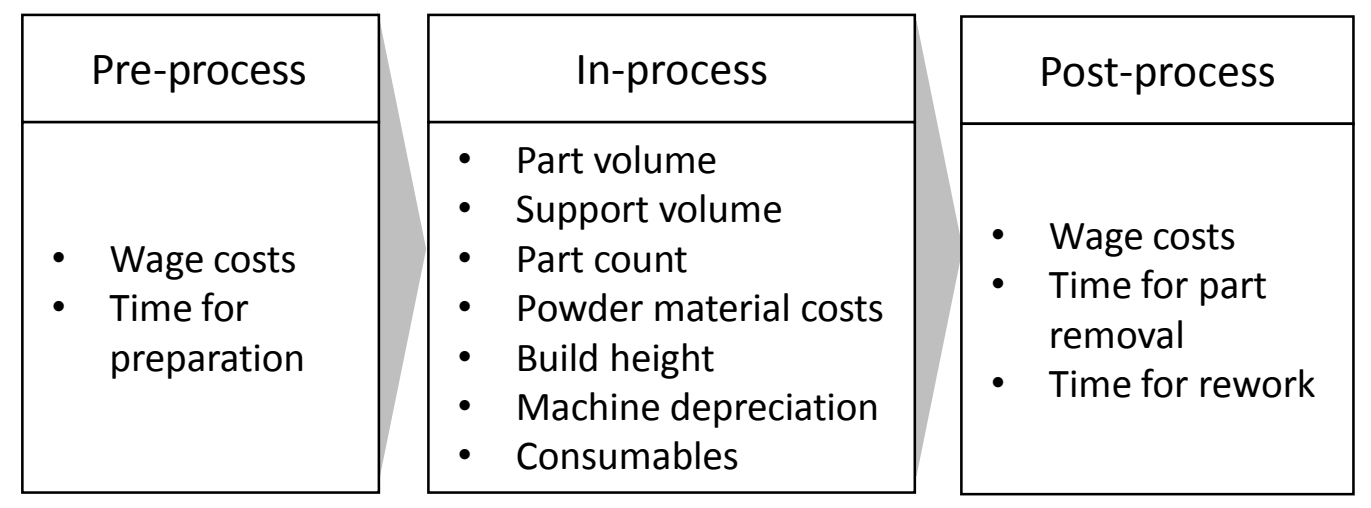

Figure 2: Cost model of the L-PBF process

The calculation of the manufacturing costs $c_{\text {total }}$ for parts produced with the L-PBF process is based on the cost model by Kranz (2017):

$$
c_{\text {total }}=c_{\mathrm{p}}+c_{m}
$$

Where $c_{\mathrm{p}}$ are the production costs and $c_{\mathrm{m}}$ are the material costs. The material costs are calculated subsequently:

$$
c_{\mathrm{m}}=\left(V_{\text {part }}+V_{\text {support }}\right) \cdot \rho \cdot c_{\text {powder }} \cdot k_{\mathrm{v}}
$$

With the volumes of the part $V_{\text {part }}$ and the supporting structure $V_{\text {support }}$, the density of the component $\rho$ the powder material costs $c_{\text {powder }}$ and a factor $k_{\mathrm{v}}$, which considers the losses of the excess, non-melted powder during recycling. The manufacturing costs consist of the costs for preprocessing $c_{\text {pre }}$ the actual manufacturing process $c_{\text {process }}$ and the post-processing $c_{\text {post }}$ of the components:

$$
c_{\mathrm{p}}=c_{\text {pre }}+c_{\text {process }}+c_{\text {post }}=c_{\text {pre }}+c_{\mathrm{m}, \mathrm{h}} \cdot t_{\text {process }}+c_{\text {post }}
$$

The pre-process includes wages for machine setup und preparation. The costs for the process itself is determined by $c_{\mathrm{m}, \mathrm{h}}$ which denotes the hourly machine costs and includes the machine depreciation and the costs for energy, inert gas and consumables.

The duration of the process itself $t_{\text {process }}$ is made up of the time for coating $t_{\mathrm{co}}$, the time for exposure $t_{\mathrm{ex}}$ of the individual layers and the number of layers $n$ :

$$
t_{\text {process }}=\left(t_{\mathrm{co}}+t_{\mathrm{ex}}\right) \cdot n
$$

The number of layers is dependent on the part height, which results from the geometry and the orientation of the component in the building chamber. The costs for the post-process includes wages and consumables for separating the parts from the building platform, the rework and the removal of the support structure.

The time required by the system for coating with new powder is independent of the number of components that are produced simultaneously. In addition, there are costs for the preparation of the system and the subsequent separation of the components, which also do not depend on the number of parts. Therefore, even with additive manufacturing, the manufacturing costs are dependent on the number of pieces to be produced. The following sample calculations are based on data from an EOS M 
290 PBF system. In the example shown in Figure 3, the manufacturing costs of the component are $€ 670$ to produce just one component and $€ 294$ per component for the production of five components.

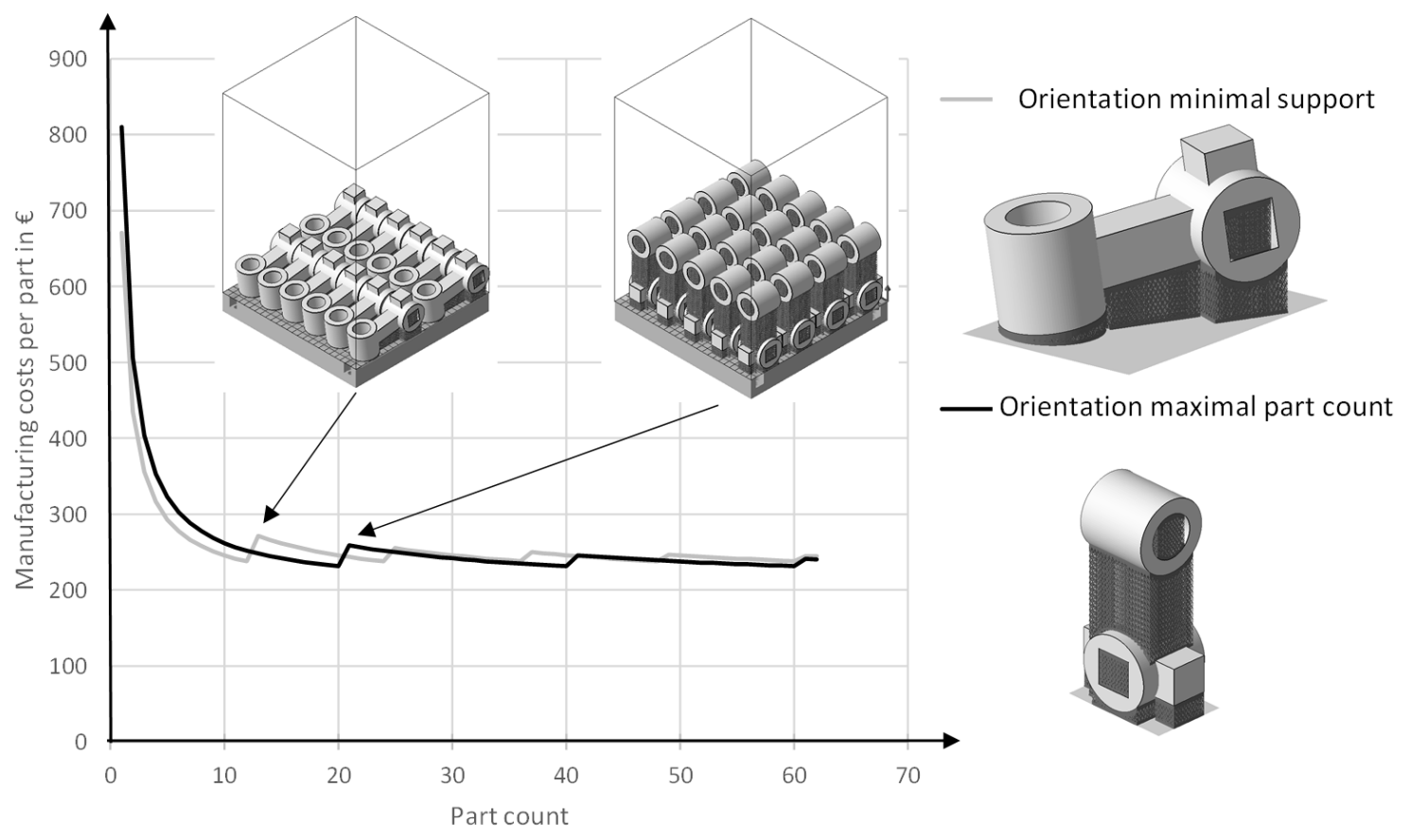

Figure 3: Manufacturing costs depending on part count and orientation

Figure 3 also shows the dependency of the manufacturing costs to the orientation of the components. Firstly, the orientation influences the amount of supporting structures necessary for the manufacturing process. Secondly, it influences the height of the component in the building space and therefore the number of layers necessary, and thirdly, the orientation of the component influences the number of parts that can be manufactured simultaneously. Figure 3 demonstrates the costs for two different orientations of the same component. The first, horizontal orientation is characterized by the minimum required volume of supporting structures and for the other orientation the number of simultaneously manufacturable components was maximized. As shown, different orientations can lead to the lowest manufacturing costs per component, depending on the total number of components to be manufactured.

The jumps in costs shown in Figure 3. occur when a new platform is required. In this example, twelve or 20 identical components fit on one platform, so that the costs increase from the 13th or 21st component onwards because then a second manufacturing process has to be carried out. Figure 3 also shows the orientation and arrangement of the example part in the two orientations on a virtual building platform. The volume of the supporting material and the height of the component in the vertical orientation is higher, which leads to significantly higher manufacturing costs for small quantities but is the more economical solution for larger quantities.

It is therefore necessary to first carry out an orientation of the resulting parts in order to evaluate the cutting variants. This then enables the calculation of the manufacturing costs of the individual parts and thus the cutting variant, in order to finally select a suitable one.

\section{PROCEDURE FOR PART SEPARATION}

As shown above, the separation of components for additive manufacturing can offer advantages over the manufacturing of a single complex component. The starting point of the procedure is a component that has already been designed with existing approaches of the DfAM. In doing so, existing methods, such as the topology optimization, the strategy of the one-piece machine (Ehrlenspiel, 1985) or the consolidation of individual components (Nie et al., 2020) are used to achieve the highest possible degree of consolidation. The aim of the following procedure is to minimize production costs by separating components. 
Since the number of components to be produced influences the manufacturing costs of additive manufacturing, this must also be considered when choosing the orientation and the resulting arrangement on the building platform. In general, the number of parts that fit on a platform depends on the size of the parts. Especially for complex components, the building chamber of the system can be better utilized if they are separated into individual parts so that more components fit on the platform with a lower degree of consolidation. This factor is also taken into account while calculating the manufacturing costs in the following procedure for component separation.

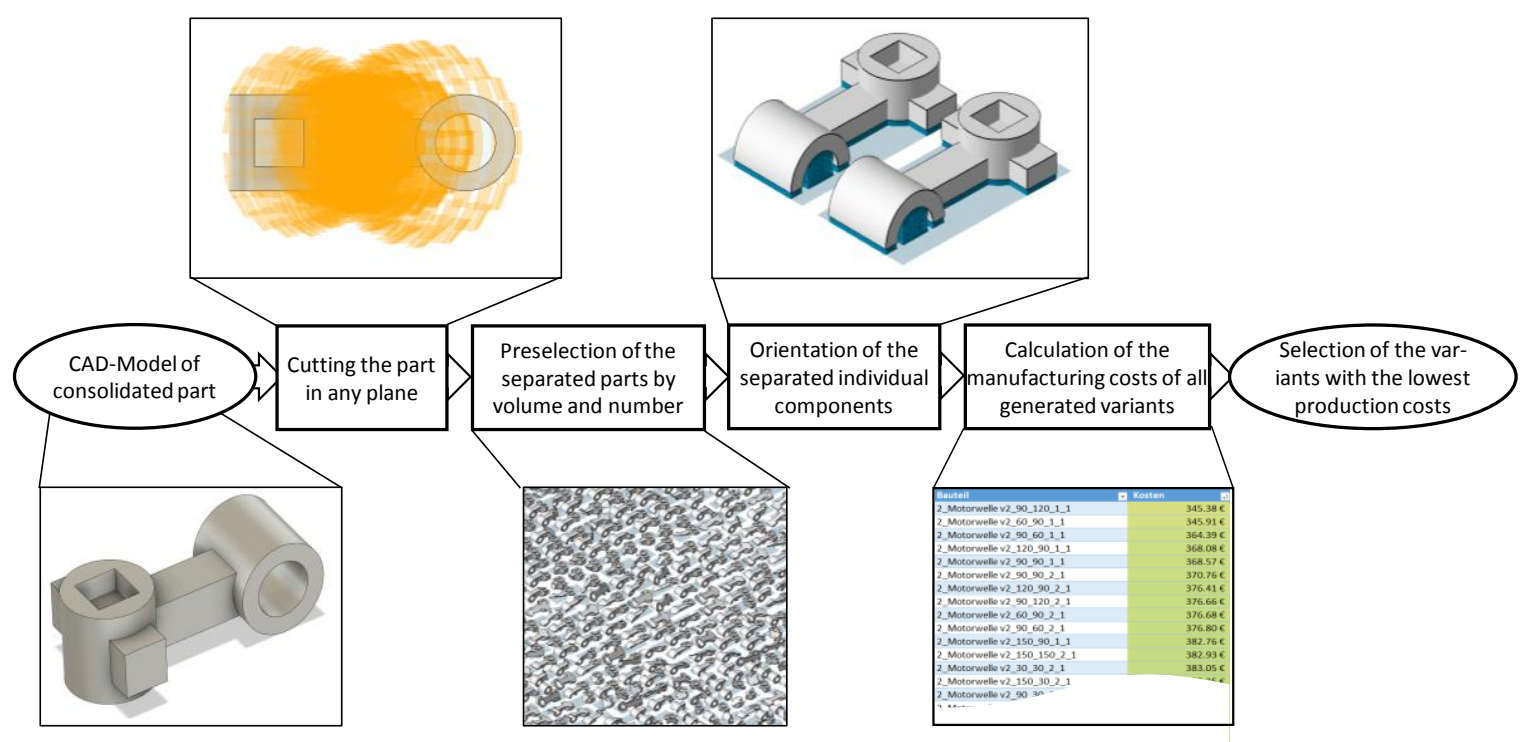

Figure 4: Procedure of component separation using an example component

Figure 4 shows the procedure using a simple example component. First, sectional planes are generated from the original, non-separated component at different angles and positions. Then by cutting along the planes the variants are created, oriented and finally selected based on the manufacturing costs. The two crucial steps are the creation of the planes and the orientation of the variants, which are described in more detail in the following.

\subsection{Part separation}

The procedure for component separation comprises the steps shown in figure 4 . The consolidated component is cut in predetermined section planes. These cutting planes are determined through a Fibonacci sphere which provides almost equally distributed points on the surface of a sphere. The vectors from the origin of the coordinate system to these points forming the normal vectors of the cutting planes. Along these normal vectors, the cutting planes are then additionally shifted parallelly through the component. The creation of the planes and the separation is done fully automated by a python script using the API of an CAD software. The number of points used for the normal vectors and the number of parallel shifts can be adjusted and has a significant influence on the runtime of the program and the number of generated variants. For each of the created section planes a component separation is performed and the parts are saved as separate files. According to the geometry of the original component two or more parts can be created per cut. Depending on the step size, several thousand variants are created, from which a preselection is made in the next step.

The preselection is made based on the number of individual parts created and the volume of the individual parts. The maximum number of individual parts that can be created by one cutting plane from a component can be limited, so that, for example, only variants with up to three individual parts are considered. In addition, variants should be excluded where the resulting parts differ too much in their volume. For this purpose, the ratio of the volume of the individual parts is calculated and a limit value is defined which must not be undercut. This leads to the fact that variants where only a very small part of the original part has been separated are not considered any further. The pre-selection leads to a strongly reduced number of variants for which a build orientation is determined in the following. 
In addition to automatically generated parts, components are separated manually. In this way, it is also possible to create cuts that deviate from a simple plane. The designer can also take into account functional aspects, such as the interchangeability of wearing surfaces, during manual separation (Reichwein et al., 2021). The manually generated parts can then be oriented with the automatically generated parts and compared in terms of manufacturing costs.

\subsection{Part Orientation for minimizing manufacturing costs}

The part orientation is an important aspect in the additive manufacturing process. It strongly influences the amount of support structure, the time needed for the build process, the number of equal parts, which can be manufactured at the same time and the surface quality of the parts. Since the choice of orientation of a component also depends on the number of components to be produced, this must be known for the following calculations. All other data for the calculation of the manufacturing costs can be calculated directly from the geometric models or are manufacturing system- or materialspecific. A calculation of the manufacturing costs for predefined orientations of the single parts follows. Like the component separation, this is done automatically by a script by means of a stepwise rotation of the individual parts according to build orientation vectors derived from a Fibonacci sphere. Based on these results, the most cost-effective orientation can be selected. In addition to minimizing the manufacturing costs, other criteria such as minimizing the volume of the supporting structures or the manufacturing time can be aimed at.

After the individual parts are oriented, the remaining variants are evaluated regarding their manufacturing costs. For this purpose, the manufacturing costs of the individual parts are added up and a comparison with the manufacturing costs of the non-separated component is made. Here it must be estimated, how the connection of the individual parts takes place after the additive manufacturing process and which costs the assembly causes, in order to make a final decision. Primarily cohesive connections are suitable, which enable the connection of the individual parts without further connecting elements and with simultaneous high strength. The choice of the joining technique strongly depends on the material used, the type of component and the available technologies and will not be considered further at this point.

\section{EVALUATION}

Figure 5 shows the motor shaft of the electrical engine of a Formula Student racing car. This part will be used as an evaluation example of the presented procedure. The structure has already been designed to minimize the mass of the aluminium component and therefore its inertia, which is important because of high accelerations during the product use. To achieve a low mass the component is designed hollow.
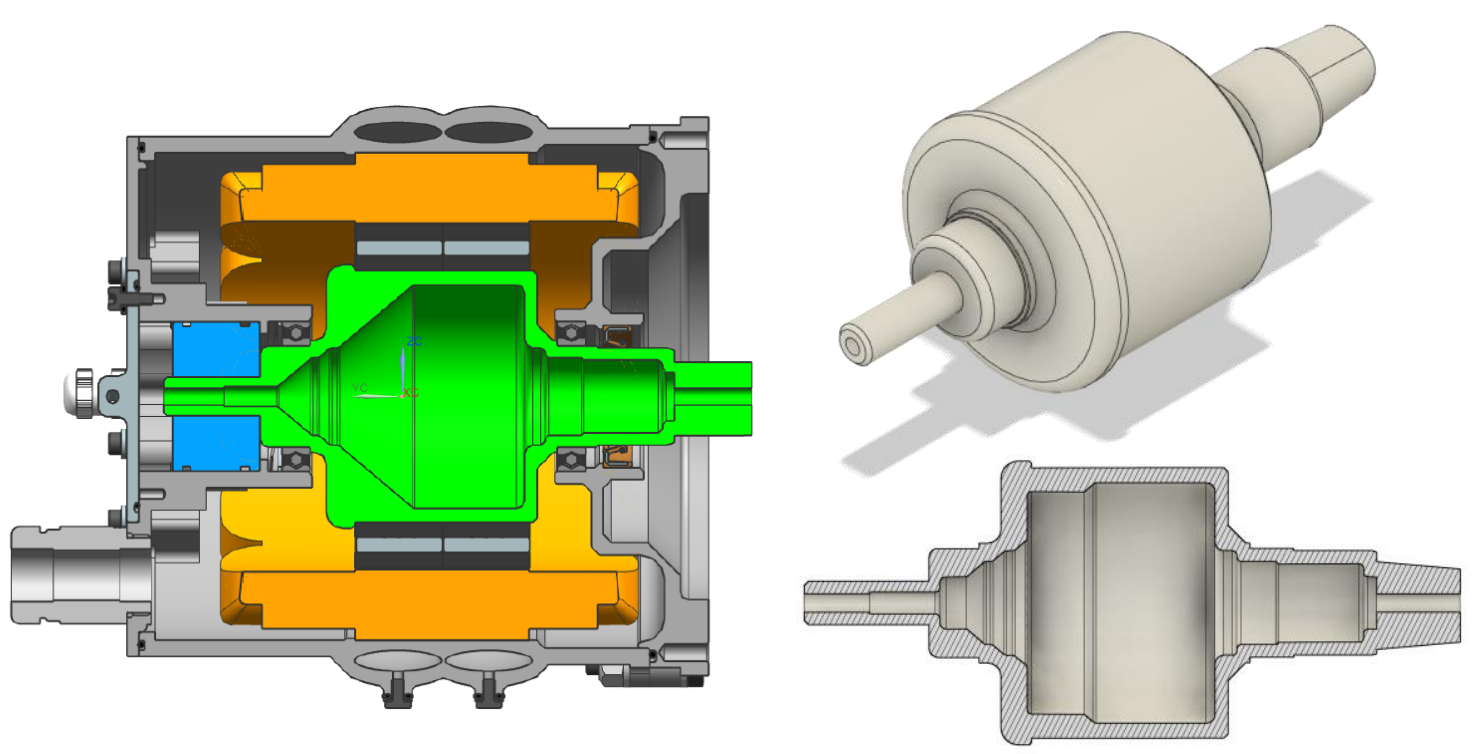

Figure 5: Electrical engine (left) and motor shaft in detail and section (right) 
During the orientation of the component on the building platform in pre-processing (see Figure 6), it becomes apparent that a remarkably high volume of supporting structures is necessary. In particular, there are support structures necessary inside of the motor shaft. These support structures cannot be removed or can only be removed at great expense and should therefore be avoided. Figure 6 shows the optimal orientation of the consolidated motor shaft to produce one part and for the production of 25 parts. It can be seen, that to produce 25 components, an orientation that allows more components on the platform is preferable for economic reasons.

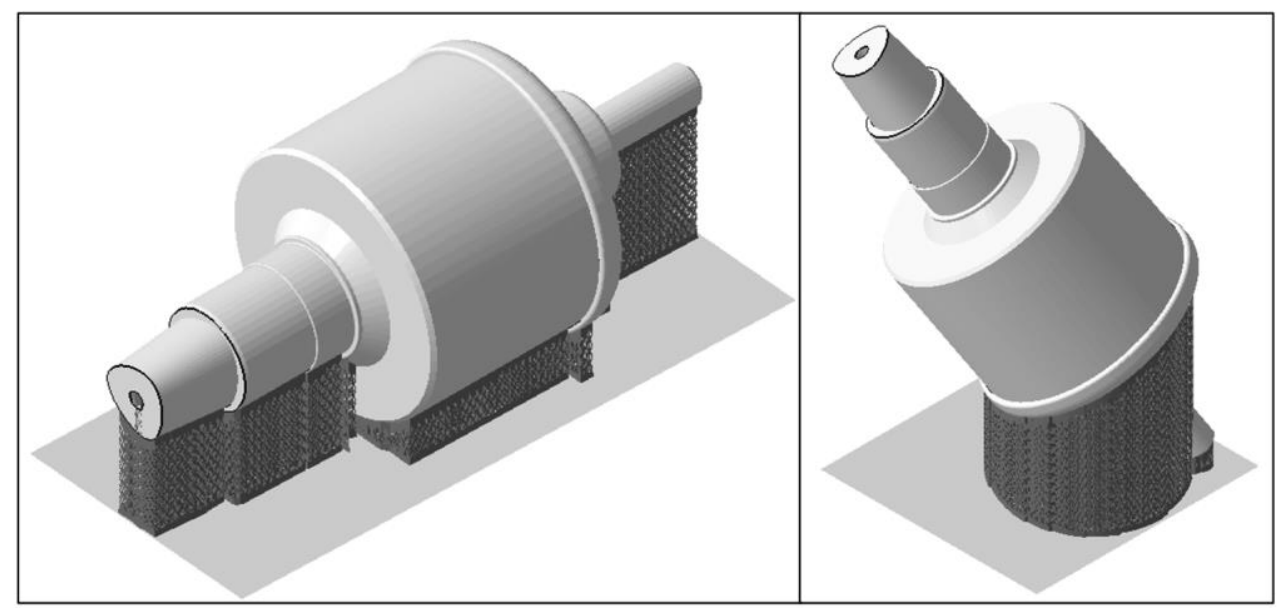

Figure 6: Motor shaft in optimal orientation for part count 1 (left) and 25 (right)

In order to reduce the overall amount of support structures, the component was separated. For this purpose, the presented procedure for component separation was applied and different alternatives were compared with regard to manufacturing costs. The separation of the component is carried out at the predefined cutting planes. During pre-selection only component separations were considered which lead to maximum 4 individual parts. The ratio of the volume of the smallest individual part to the total volume must not fall below $10 \%$. With these boundary conditions and a parallel shift of the planes by $20 \mathrm{~mm}$ each the separation results in 154 variants for the motor shaft.

Subsequently, an optimal orientation with regard to the manufacturing costs is calculated for each individual part. This enables the evaluation of the separation variants in order to select the variant with the lowest manufacturing costs for the required number of components. The computation of the separations and the subsequent evaluation of the parts took about 3 minutes for this example. In addition to the manufacturing costs, the planes can also be evaluated and selected based on criteria such as the volume of the supporting structures, the duration of the manufacturing process or how many identical components can be manufactured on one platform. For example, the separation level can also be selected to ensure the shortest possible production time.

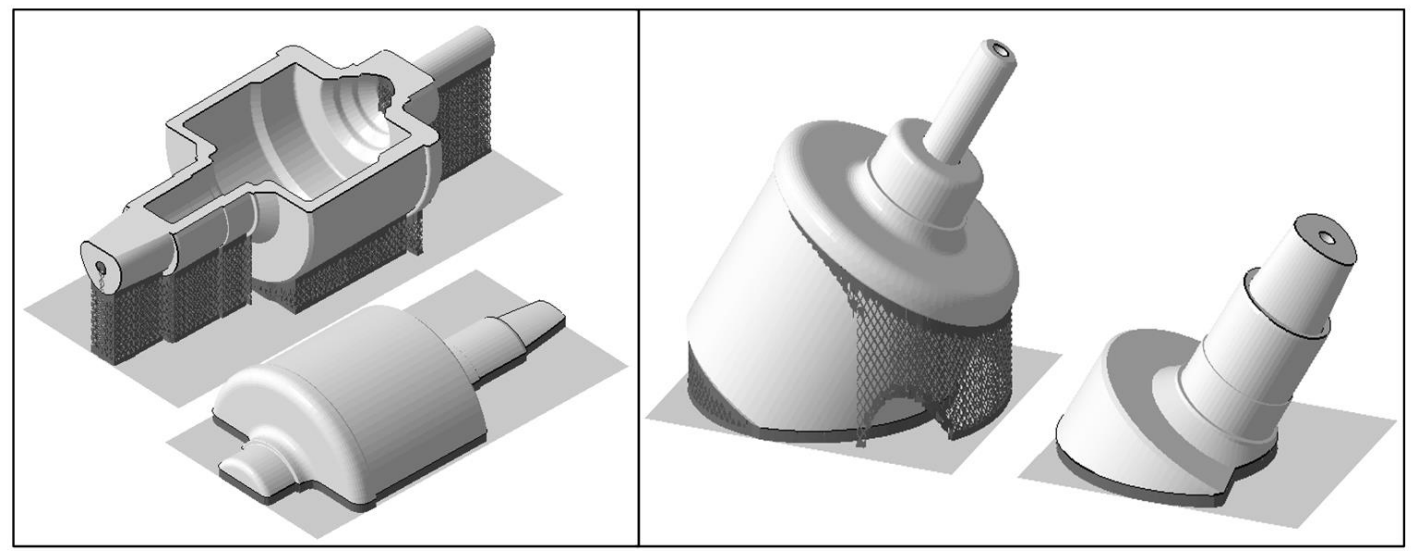

Figure 7: Separated motor shaft, optimal cutting plane for part count 1 (left) and 25 (right)

Figure 7 shows the results of the performed component separation. To produce 25 components, the manufacturing costs of the separated motor shaft were reduced by $33 \%$ in contrast to the consolidated 
design. This results from the reduction of support structure. Additionally, the separation of the motor shaft enables the removal of support structures inside of the hollow component before joining them.

In addition to the motor shaft the procedure was evaluated with the mounting of the steering shaft of the same race car. For the mounting a topology optimization was carried out to minimize the mass. This results in a complex geometry and a great amount of support structure to attach overhanging surfaces to the build platform. Another disadvantage of the complex geometry is the low usage of the building space. Only two mountings can be manufactured on the build platform of the EOS M290 PBF system. The separation of the mounting leads to even greater savings and the manufacturing costs could be reduced by $54 \%$. The economically best solution is the separation of the component in three single parts as depicted in Figure 8. Here the main reason for the savings is the orientation of the individual parts, which allows a higher use of the building chamber volume. In contrast to the consolidated design that only allows two mountings in one manufacturing process.

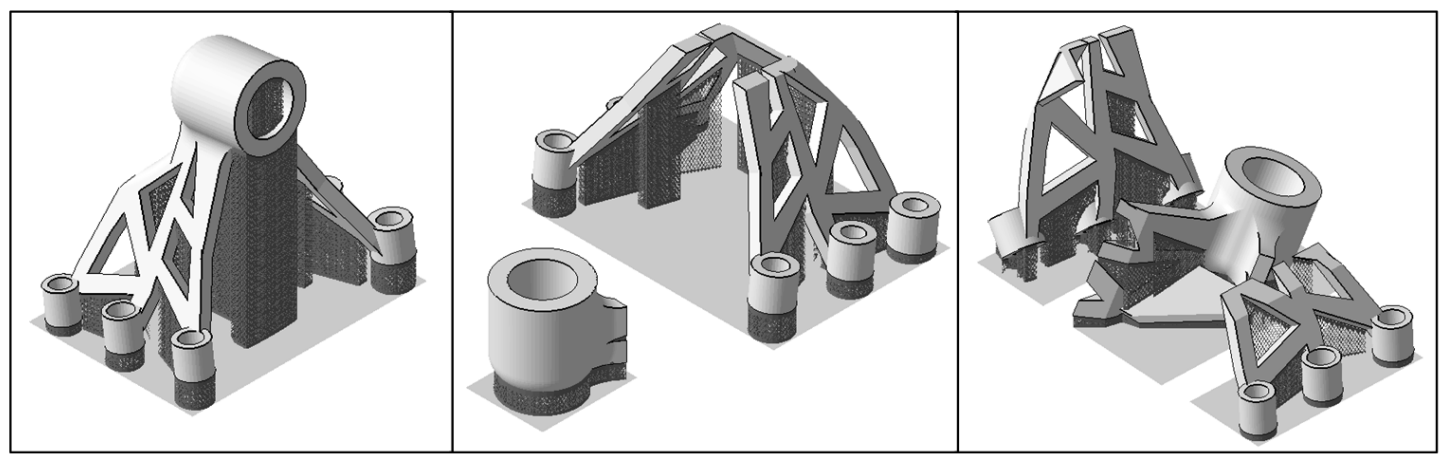

Figure 8: Steering shaft bearing consolidated part (left), separated in 2 parts (middle) and separated in 3 parts (right)

Table 1 gives an overview of the manufacturing costs of the presented variants for the motor shaft and the mounting of the steering shaft. The costs for the connection of the separated parts are not considered yet. These are strongly dependant on the chosen assembly process, material, etc. The presented procedure allows a well-founded decision on the separation of components, but this decision has to be made for each individual case. Suitable manufacturing technologies for the above presented examples are diffusion welding and laser welding, as it allows the aluminium components to be joined together without the material characteristics in the joint deviating significantly from those of the base material, thus ensuring that the mechanical forces of the component are transmitted.

Table 1: Overview of manufacturing costs of consolidated and separated components

\begin{tabular}{l|l|l}
\multirow{2}{*}{ Description } & \multicolumn{2}{|l}{ Manufacturing costs } \\
\cline { 2 - 3 } & Part count 1 & Part count 25 \\
\hline
\end{tabular}

\begin{tabular}{l|l|l} 
Motor shaft \\
\hline Consolidated part & $€ 844$ & $€ 513$ \\
\hline Separated in two parts & $€ 1041$ & $€ 345$ \\
\hline
\end{tabular}

\begin{tabular}{l|l|l} 
Steering shaft bearing & \\
\hline \hline Consolidated part & $€ 1102$ & $€ 1029$ \\
\hline Separated in two parts & $€ 1264$ & $€ 586$ \\
\hline Separated in three parts & $€ 1547$ & $€ 484$
\end{tabular}

\section{SUMMARY AND OUTLOOK}

The presented procedure enables the automated determination of a separation plane in order to optimize the additive manufacturing of complex parts or components that are larger than the available space with respect to various criteria. Thus, the procedure complements the general efforts of the DfAM to consolidate components under special consideration of the manufacturing restrictions. By 
means of an example it was shown that by separating a complex component a reduction of manufacturing costs, process time, volume of the supporting structure and number of components to be manufactured simultaneously can be achieved.

After the additive manufacturing of the individual parts, they have to be joined again. For this purpose, cohesive component connections are particularly suitable, such as various welding processes (diffusion and ultrasonic welding) and, especially for plastic components, adhesive bonding processes. The design and dimensioning of the joints will therefore be the subject of future investigations.

Currently, only cuts based on planes are performed and more complex cut geometries are neglected. This also still offers potential for improvements in the procedure. The already mentioned possibility to manually separate the part in addition to the automatically performed part separation can be supported by a methodical approach. Thus, aspects such as the exchangeability of certain elements that are subject to wear during operation can be taken into account.

\section{REFERENCES}

Adam, G.A.O. (2015), Systematische Erarbeitung von Konstruktionsregeln für die additiven Fertigungsverfahren Lasersintern, Laserschmelzen und Fused Deposition Modeling, Paderborn, Diss., Shaker, Aachen.

Deka, A. and Behdad, S. (2019), "Part Separation Technique for Assembly-Based Design in Additive Manufacturing using Genetic Algorithm”, Procedia Manufacturing, Vol. 34, pp. 764-771.

Ehrlenspiel, K. (1985), Kostengünstig konstruieren: Kostenwissen, Kosteneinflüsse, Kostensenkung; Konstruktionsbücher, Vol. 35, Springer, Berlin.

Gibson, I., Rosen, D. and Stucker, B. (2015), Additive manufacturing technologies: 3D printing, rapid prototyping, and direct digital manufacturing, Springer, New York, NY.

Kim, S. and Moon, S.K. (2020), "A Part Consolidation Design Method for Additive Manufacturing based on Product Disassembly Complexity”, Applied Sciences, Vol. 10 No. 3, p. 1100.

Kranz, J. (2017), Methodik und Richtlinien für die Konstruktion von laseradditiv gefertigten Leichtbaustrukturen, Springer, Berlin, Heidelberg.

Kranz, J., Herzog, D. and Emmelmann, C. (2015), "Design guidelines for laser additive manufacturing of lightweight structures in TiAl6V4", Journal of Laser Applications, Vol. 27 No. S1, S14001.

Krause, D. and Gebhardt, N. (2018), Methodische Entwicklung Modularer Produktfamilien: Hohe Produktvielfalt Beherrschbar Entwickeln, Vieweg, Berlin, Heidelberg.

Kumke, M. (2018), Methodisches Konstruieren von additiv gefertigten Bauteilen, Springer Fachmedien Wiesbaden, Wiesbaden.

Laverne, F., Segonds, F., Anwer, N. and Le Coq, M. (2015), “Assembly Based Methods to Support Product Innovation in Design for Additive Manufacturing: An Exploratory Case Study", Journal of Mechanical Design, Vol. 137 No. 12, p. 121701.

Nie, Z., Jung, S., Kara, L.B. and Whitefoot, K.S. (2020), “Optimization of Part Consolidation for Minimum Production Costs and Time Using Additive Manufacturing”, Journal of Mechanical Design, Vol. 142 No. 7.

Oh, Y., Zhou, C. and Behdad, S. (2018), "Part decomposition and 2D batch placement in single-machine additive manufacturing systems", Journal of Manufacturing Systems, Vol. 48, pp. 131-139.

Reichwein, J., Kaspar, J., Vielhaber, M. and Kirchner, E. (2019), "Exploitation of AM-Potentials by Linking Manufacturing Processes to Function-Driven Product Design", Proceedings of the Design Society: International Conference on Engineering Design, Vol. 1 No. 1, pp. 739-748.

Reichwein, J., Rudolph, K., Geis, J., Kirchner, E. (2021), "Adapting Product Architecture to Additive Manufacturing through Consolidation and Separation", Procedia CIRP: CIRP Design conference 2021.

Schulte, F.; Würtenberger, J.; Steffan, K.-E.; Kirchner, (2020), "TRIZ als Schlüssel zu den Potentialen additiver Fertigungsverfahren", In: E. Lachmayer, R.; Lippert, R. B., Kaierle, S. (Hrsg.) Konstruktion für die additive Fertigung 2018, S. 55-75, Berlin, Heidelberg, Springer Vieweg, ISBN 978-3-662-59057-7.

Wohlers, T.T. (2018), Wohlers Report: $3 d$ printing and additive manufacturing state of the industry, Wohlers Associates, Fort Collins. 